УДК 517.9

\title{
On Applications of the Cayley Graphs of some Finite Groups of Exponent Five
}

\author{
Alexander A. Kuznetsov* \\ Konstantin V.Safonov ${ }^{\dagger}$ \\ Institute of Computer Science and Telecommunications \\ Reshetnev Siberian State University of Science and Technology \\ Krasnoyarsky Rabochy, 31, Krasnoyarsk, 660037
}

Russia

Received 17.04.2017, received in revised form 20.04.2017, accepted 16.10.2017

Let $B_{0}(2,5)$ be the largest two-generator finite Burnside group of exponent five. It has the order $5^{34}$. We define an automorphism $\varphi$ which translates generating elements into their inverses. Let $C_{B_{0}(2,5)}(\varphi)$ be the centralizer of $\varphi$ in $B_{0}(2,5)$. It is known that $\left|C_{B_{0}(2,5)}(\varphi)\right|=5^{16}$. The growth functions of the centralizer are computed for some generating sets in the article. As the result we got diameters and average diameters of corresponding the Cayley graphs of $C_{B_{0}(2,5(\varphi)}$.

Keywords: periodic group, collection process, Hall's polynomials, the Cayley graph, multiprocessor computer system.

DOI: $10.17516 / 1997-1397-2018-11-1-70-78$.

One of the important tools for defining the structure of a group is the study of its growth with respect to a fixed generating set. Let $G=\langle X\rangle$. We call the ball $K_{s}$ of radius $s$ of a group $G$ the set of all its elements, which can be presented as a group words with length $s$ in the alphabet $X$. For each nonnegative integer $s$, one can define the growth function of the group $F(s)$, which is equal to the number of elements of the group $G$ with respect to $X$ that can be represented as an irreducible group words with the length $s$. Thus,

$$
F(0)=\left|K_{0}\right|=1, F(s)=\left|K_{s}\right|-\left|K_{s-1}\right| \text { when } s \in \mathbb{N} .
$$

As a rule, the growth function of a finite group is represented in the form of a table which contains non-zero values of $F(s)$.

Unfortunately, although the computation of the growth function of a large finite group is solvable, it is a rather complicated problem. This is due to the fact that, in general, the task of determination of the minimal word of a group element, as shown by S. Iven and O. Goldreich [1], is $\mathrm{NP}$-hard. Thus, in the worst case, the number of elementary operations that must be performed to solve this problem is an exponential function of $|X|$.

Note also that computing the growth function of a group, we define in a parallel way the characteristics of the corresponding Cayley graph, for instance, the diameter and the average diameter [2]. Let $F\left(s_{0}\right)>0$, but $F\left(s_{0}+1\right)=0$, then $s_{0}$ will be the diameter of the Cayley graph of the group $G$ in the generating alphabet $X$, which we will denote $D_{X}(G)$. Accordingly, the average diameter $\bar{D}_{X}(G)$ is equal to $\frac{1}{|G|} \sum_{s=0}^{s_{0}} s \cdot F(s)$.

\footnotetext{
*alex_kuznetsov80@mail.ru

†safonovkv@rambler.ru

(C) Siberian Federal University. All rights reserved
} 
In recent decades, the Cayley graph theory has developed as a separate large branch of the graph theory. The Cayley graphs are used both in mathematics and outside it. In particular, the Cayley graphs were used in information technology after the pioneering work of 1986 by S. Akers and B. Krishnamurti [3], who first proposed the use of these graphs to represent computer networks, including for topology modeling (i. e., methods of connecting processors to each other) multiprocessor computer systems (MCS) - supercomputers, as well as data centers [4,5]. Since then this direction is actively developing. This is due to the fact that the Cayley graphs have many attractive properties, of which we distinguish their regularity, vertex transitivity, small diameter and degree with a sufficiently large number of vertices in the graph. Note that such basic network topologies as «ring», «hypercube» and «torus» are the Cayley graphs. According to the latest data, the performance of the most powerful MCS approaches 100 petaFLOPS, with the total number of cores in the processors already exceeding 10 millions. As you know, the network topology is a critical parameter of the MCS performance. Therefore, there is a reason to believe that, not in the far future, the knowledge on very large graphs will be needed in the designing of distributed systems, in which peak performance will reach 1 exaFLOPS and above.

One of the widely used topologies of MCS is the $k$-dimensional hypercube. This graph is determined by the group $B(k, 2)$, which is the $k$-generated Burnside group of period 2. $B(k, 2)$, has a simple structure and is equal to the direct product of $k$ copies of a cyclic group of order 2 . In the work [6], the Cayley graphs of the groups $B(k, 3)$, i. e. groups of period 3 , were studied and the comparative analysis of these graphs with respect to a hypercube carried out. The analysis showed that the characteristics of $B(k, 3)$ are more preferable than characteristics of $B(k, 2)$. It means that, while paired comparison graphs $B\left(k_{1}, 3\right)$ and $B\left(k_{2}, 2\right)$ with approximately the same number of vertices, the first ones have the smaller diameters, the average diameters and the degrees. A similar result was obtained in [7] in the study of groups of period 4. In this regard, the task of the study of the Cayley graphs of finite Burnside groups of other periods is interesting.

Let $B_{0}(2,5)=\left\langle a_{1}, a_{2}\right\rangle$ be a maximal finite two-generated Burnside group of period 5 , which order is equal to $5^{34}[8]$. Using the computer algebra system GAP, it is easy to obtain pcpresentation (power commutator presentation) of this group [4,9]. In this case, each element $g \in B_{0}(2,5)$ can be uniquely written in the following form:

$$
\forall g \in B_{0}(2,5) \Rightarrow g=a_{1}^{\alpha_{1}} \cdot a_{2}^{\alpha_{2}} \cdot \ldots \cdot a_{34}^{\alpha_{34}}, \alpha_{i} \in \mathbb{Z}_{5}, i=1,2, \ldots, 34
$$

Here $a_{1}$ and $a_{2}$ are the generating elements $B_{0}(2,5), a_{3}, \ldots, a_{34}$ are the commutators, which are computed recursively by $a_{1}$ and $a_{2}[8]$.

In addition to the applied interest, there is another reason for the increased attention of researchers to the growth function of $B_{0}(2,5)$. This is because an obtained information may be useful in solving of the open problem on finiteness of $B(2,5)$ which is a free two-generated Burnside groups of period 5. If $B(2,5)$ is finite, then $B_{0}(2,5)=B(2,5)$. However, in the foreseeable future, to calculate the growth function of $B_{0}(2,5)$ is hardly possible, since the number of elements is very large:

$$
5^{34}=582076609134674072265625 \approx 5 \cdot 10^{23} .
$$

Note that until now, with the help of computer calculations, it was possible to obtain the growth functions of the factor-groups of the group $B_{0}(2,5)$, which order does not exceed $5^{17}[10]$. 
Let us consider the map $\varphi$ of the following form:

$$
\varphi:\left\{\begin{array}{l}
a_{1} \rightarrow a_{1}^{-1} \\
a_{2} \rightarrow a_{2}^{-1}
\end{array}\right.
$$

It is easy to see [11], that $\varphi$ is an involutive automorphism of groups $B(2,5)$ and $B_{0}(2,5)$.

Let $C_{B(2,5)}(\varphi)$ and $C_{B_{0}(2,5)}(\varphi)$ be the centralizers of the automorphism $\varphi$ in $B(2,5)$ and $B_{0}(2,5)$ respectively. By the theorem of V. P. Shunkov [12], if $C_{B(2,5)}(\varphi)$ is a finite group, then the group $B(2,5)$ is also finite. In other words, if $C_{B(2,5)}(\varphi)=C_{B_{0}(2,5)}(\varphi)$, then $B(2,5)=B_{0}(2,5)$. By this reason, the study of the growth of $C_{B_{0}(2,5)}(\varphi)$ is of great interest. Further, for brevity, we will write $C$ instead of $C_{B_{0}(2,5)}(\varphi)$.

In [11], the structure of group $C$ is studied and the following results are obtained:

1. $|C|=5^{16}$,

2. $C=C_{0} \times\langle z\rangle$ where $\left|C_{0}\right|=5^{15}$ and $|\langle z\rangle|=5$;

3. $z$ is the central element of the group $B_{0}(2,5)$,

4. $C_{0}=\left\langle X_{0}\right\rangle$ where $\left|X_{0}\right|=4$ is the minimal number of the generators of $C_{0}$,

5. pc-presentation of $C_{0}$ is computed.

The purpose of this article is the study of the growth function of the group $C$ with respect to the minimal generating set $X=X_{0} \cup\{z\}$, and also the symmetric $-Y=X \cup X^{-1}$.

Since for the evaluation of growth function it is required to multiply elements of the group, then for the practical implementation an efficient algorithm for multiplication is needed. There are two ways to calculate the product of elements in the group given by pc-presentation: the collective process $[4,9]$ and Hall's polynomials method [13]. Numerous computational experiments showed that the second method allows to multiply the elements in these groups much faster than in a collecting process (at least in exponent) $[6,10,14,15]$.

The documentation of the computer algebra system GAP refers to the possibility of automated calculation of Hall's polynomials in the simplest cases. However, in the general case, this task is not trivial, because is not reduced to routine computation and requires the involvement of programming languages that support complex regular expressions, and also systems of computer mathematics with a wide range of procedures for symbolic computation. In fact, working with a group that has a large order, usually it is required the unique revision of the code that takes into account the feature of the group structure and characteristics of the computer.

In the following section of the article we present an algorithm to calculate the Hall's polynomials of the group $C_{0}$. This algorithm in general was implemented in $\mathrm{C}++$, with the exception of symbolic procedures, which were written in the MATLAB language.

In the last section, the results of computer calculations of the growth function of the group $C$ with respect to the generating set $X$ and $Y$ are given.

\section{The Hall's polynomials of the group $C_{0}$}

Let $C_{0}=\left\langle X_{0}\right\rangle$ where $X_{0}=\left\{a_{1}, a_{2}, a_{3}, a_{4}\right\}$ is the minimal generating set of $C_{0}$. The following theorem is proved.

Theorem. Let $a_{1}^{x_{1}} \ldots a_{15}^{x_{15}}$ and $a_{1}^{y_{1}} \ldots a_{15}^{y_{15}}$ be two arbitrary elements of the group $C_{0}$ recorded in the commutator form. Then their product is equal $a_{1}^{x_{1}} \ldots a_{15}^{x_{15}} \cdot a_{1}^{y_{1}} \ldots a_{15}^{y_{15}}=a_{1}^{z_{1}} \ldots a_{15}^{z_{15}}$ where $z_{i} \in \mathbb{Z}_{5}$ are Hall's polynomials given by formulas (1-15). 


$$
\begin{gathered}
z_{1}=x_{1}+y_{1}, \\
z_{2}=x_{2}+y_{2}, \\
z_{3}=x_{3}+y_{3} \\
z_{4}=x_{4}+y_{4} \\
z_{5}=x_{5}+y_{5}+x_{2} y_{1}+4 x_{3} y_{2}, \\
z_{6}=x_{6}+y_{6}+x_{3} y_{1}+4 x_{3} y_{2}, \\
z_{7}=x_{7}+y_{7}+x_{4} y_{1}, \\
z_{8}=x_{8}+y_{8}+x_{4} y_{2}, \\
z_{9}=x_{9}+y_{9}+x_{4} y_{3} \\
z_{11}=x_{11}+y_{11}+4 x_{3} y_{2}+2 x_{4} y_{2}+x_{8} y_{4}+3 x_{4}^{2} y_{2}+x_{4} y_{2} y_{4}, \\
z_{12}=x_{12}+y_{12}+2 x_{3} y_{2}+2 x_{4} y_{3}+x_{9} y_{4}+3 x_{4}^{2} y_{3}+x_{4} y_{3} y_{4}, \\
z_{13}=x_{13}+y_{13}+3 x_{3} y_{2}+2 x_{4} y_{1}+2 x_{5} y_{4}+x_{7} y_{2}+4 x_{8} y_{1}+2 x_{7} y_{4}+x_{10} y_{4}+ \\
+2 x_{4}^{2} y_{1}+x_{4}^{3} y_{1}+3 x_{7} y_{4}^{2}+3 x_{4} y_{1} y_{4}^{2}+3 x_{4}^{2} y_{1} y_{4}+2 x_{2} x_{4} y_{1}+2 x_{2} y_{1} y_{4}+x_{4} y_{1} y_{2}+4 x_{4} y_{1} y_{4}, \\
z_{14}=x_{14}+y_{14}+3 x_{3} y_{2}+2 x_{4} y_{2}+4 x_{6} y_{4}+2 x_{7} y_{3}+3 x_{9} y_{1}+2 x_{8} y_{4}+x_{11} y_{4}+ \\
+2 x_{4}^{2} y_{2}+x_{4}^{3} y_{2}+3 x_{8} y_{4}^{2}+3 x_{4} y_{2} y_{4}^{2}+3 x_{4}^{2} y_{2} y_{4}+4 x_{3} x_{4} y_{1}+4 x_{3} y_{1} y_{4}+2 x_{4} y_{1} y_{3}+4 x_{4} y_{2} y_{4}, \\
z_{15}=x_{15}+y_{15}+x_{3} y_{2}+2 x_{4} y_{3}+x_{8} y_{3}+4 x_{9} y_{2}+2 x_{9} y_{4}+x_{12} y_{4}+2 x_{4}^{2} y_{3}+ \\
+x_{4}^{3} y_{3}+3 x_{9} y_{4}^{2}+3 x_{4} y_{3} y_{4}^{2}+3 x_{4}^{2} y_{3} y_{4}+2 x_{3} x_{4} y_{2}+2 x_{3} y_{2} y_{4}+x_{4} y_{2} y_{3}+4 x_{4} y_{3} y_{4}
\end{gathered}
$$

Proof. Let's write the commutator representation of the group $C_{0}$ [11]: $a_{1}, a_{2}, a_{3}, a_{4}$ - generators;

$a_{5}=\left[a_{2}, a_{1}\right], a_{6}=\left[a_{3}, a_{1}\right], a_{7}=\left[a_{4}, a_{1}\right], a_{8}=\left[a_{4}, a_{2}\right], a_{9}=\left[a_{4}, a_{3}\right]-$ commutators of weight $2 ;$ $a_{10}=\left[a_{4}, a_{1}, a_{4}\right], a_{11}=\left[a_{4}, a_{2}, a_{4}\right], a_{12}=\left[a_{4}, a_{3}, a_{4}\right]$ - commutators of weight 3 ;

$a_{13}=\left[a_{4}, a_{1}, a_{4}, a_{4}\right], a_{14}=\left[a_{4}, a_{2}, a_{4}, a_{4}\right], a_{15}=\left[a_{4}, a_{3}, a_{4}, a_{4}\right]$ - commutators of weight 4.

List of defining relations $R$ for commutators: (trivial relations $\left[a_{j}, a_{i}\right]=1$ are not given):

$a_{i}^{5}=1(1 \leqslant i \leqslant 15),\left[a_{2}, a_{1}\right]=a_{5},\left[a_{3}, a_{1}\right]=a_{6},\left[a_{3}, a_{2}\right]=a_{5}^{4} a_{6}^{4} a_{10}^{2} a_{11}^{4} a_{12}^{2} a_{13}^{3} a_{14}^{3} a_{15}$, $\left[a_{4}, a_{1}\right]=a_{7},\left[a_{4}, a_{2}\right]=a_{8},\left[a_{4}, a_{3}\right]=a_{9},\left[a_{5}, a_{4}\right]=a_{13}^{2},\left[a_{6}, a_{4}\right]=a_{14}^{4},\left[a_{7}, a_{2}\right]=a_{13}$, $\left[a_{7}, a_{3}\right]=a_{14}^{2},\left[a_{7}, a_{4}\right]=a_{10},\left[a_{8}, a_{1}\right]=a_{13}^{4},\left[a_{8}, a_{3}\right]=a_{15},\left[a_{8}, a_{4}\right]=a_{11},\left[a_{9}, a_{1}\right]=a_{14}^{3}$, $\left[a_{9}, a_{2}\right]=a_{15}^{4},\left[a_{9}, a_{4}\right]=a_{12},\left[a_{10}, a_{4}\right]=a_{13},\left[a_{11}, a_{4}\right]=a_{14},\left[a_{12}, a_{4}\right]=a_{15}$.

Thus,

$$
C_{0}=\left\langle a_{1}, a_{2}, \ldots, a_{15} \mid R\right\rangle .
$$

Each element of the group is expressed uniquely as a normal commutator word:

$$
\forall g \in C_{0} \quad g=a_{1}^{x_{1}} a_{2}^{x_{2}} \ldots a_{15}^{x_{15}}, \quad x_{i} \in \mathbb{Z}_{5} .
$$

Sometimes we will write $g=\left(x_{1}, \ldots, x_{15}\right)$. 
In order to determine the functions $z_{i}$ first we need to calculate the products of $a_{j}^{y} a_{i}^{x}$ for all $1 \leqslant i<j \leqslant 15, x, y=1,2,3,4$. For the pair $(j, i)$, it is required to find the interpolation polynomial for each of the 15 commutators by the 16 values of the product $(y, x)$.

Let's start with the first pair $a_{2}^{y} a_{1}^{x}$ :

$$
\begin{array}{ll}
a_{2}^{1} a_{1}^{1}=(1,1,0,0,1,0,0,0,0,0,0,0,0,0,0), \quad a_{2}^{2} a_{1}^{1}=(1,2,0,0,2,0,0,0,0,0,0,0,0,0,0), \\
a_{2}^{3} a_{1}^{1}=(1,3,0,0,3,0,0,0,0,0,0,0,0,0,0), \quad a_{2}^{4} a_{1}^{1}=(1,4,0,0,4,0,0,0,0,0,0,0,0,0,0), \\
a_{2}^{1} a_{1}^{2}=(2,1,0,0,2,0,0,0,0,0,0,0,0,0,0), \quad a_{2}^{2} a_{1}^{2}=(2,2,0,0,4,0,0,0,0,0,0,0,0,0,0), \\
a_{2}^{3} a_{1}^{2}=(2,3,0,0,1,0,0,0,0,0,0,0,0,0,0), \quad a_{2}^{4} a_{1}^{2}=(2,4,0,0,3,0,0,0,0,0,0,0,0,0,0), \\
a_{2}^{1} a_{1}^{3}=(3,1,0,0,3,0,0,0,0,0,0,0,0,0,0), \quad a_{2}^{2} a_{1}^{3}=(3,2,0,0,1,0,0,0,0,0,0,0,0,0,0), \\
a_{2}^{3} a_{1}^{3}=(3,3,0,0,4,0,0,0,0,0,0,0,0,0,0), \quad a_{2}^{4} a_{1}^{3}=(3,4,0,0,2,0,0,0,0,0,0,0,0,0,0), \\
a_{2}^{1} a_{1}^{4}=(4,1,0,0,4,0,0,0,0,0,0,0,0,0,0), \quad a_{2}^{2} a_{1}^{4}=(4,2,0,0,3,0,0,0,0,0,0,0,0,0,0), \\
a_{2}^{3} a_{1}^{4}=(4,3,0,0,2,0,0,0,0,0,0,0,0,0,0), \quad a_{2}^{4} a_{1}^{4}=(4,4,0,0,1,0,0,0,0,0,0,0,0,0,0) .
\end{array}
$$

Let's write:

$$
a_{2}^{y} a_{1}^{x}=a_{1}^{x} a_{2}^{y} a_{3}^{f_{3}^{(1,2)}(x, y)} a_{4}^{f_{4}^{(1,2)}(x, y)} \ldots a_{15}^{f_{15}^{(1,2)}(x, y)}
$$

where $f_{r}^{(1,2)}(x, y)=\sum_{p=1}^{4} \sum_{q=1}^{4} \beta_{p q}^{r} x^{p} y^{q}$ are some polynomials over the field $\mathbb{Z}_{5}$. To find them, let's perform interpolation for each commutator $r=3,4, \ldots, 15$.

To find $f_{r}^{(1,2)}(x, y)$, it is required to solve a system of linear equations over the given field:

$$
\sum_{p=1}^{4} \sum_{q=1}^{4} \beta_{p q}^{r} x^{p} y^{q}=z_{r}^{y x} \quad \forall x, y=1,2,3,4,
$$

where $z_{r}^{y x}$ is a value of $r$-th commutator for the pair $(y, x)$. This system will have 16 variables and consist of 16 equations.

Let's show how to find $f_{5}^{(1,2)}(x, y)$ at the example of the 5 -th commutator. For short, we will write $\beta_{p q}$ instead of $\beta_{p q}^{5}$. Substituting in (16) all values of $z_{5}^{y x}$ we receive:

$$
\left[\begin{array}{llllllllllllllll}
1 & 1 & 1 & 1 & 1 & 1 & 1 & 1 & 1 & 1 & 1 & 1 & 1 & 1 & 1 & 1 \\
2 & 4 & 2 & 3 & 4 & 2 & 1 & 3 & 4 & 2 & 1 & 3 & 4 & 1 & 3 & 1 \\
3 & 4 & 3 & 2 & 4 & 3 & 1 & 2 & 4 & 3 & 1 & 2 & 4 & 1 & 2 & 1 \\
4 & 1 & 4 & 4 & 1 & 4 & 1 & 4 & 1 & 4 & 1 & 4 & 1 & 1 & 4 & 1 \\
2 & 2 & 4 & 2 & 4 & 3 & 2 & 4 & 3 & 1 & 4 & 3 & 1 & 3 & 1 & 1 \\
4 & 3 & 3 & 1 & 1 & 1 & 2 & 2 & 2 & 2 & 4 & 4 & 4 & 3 & 3 & 1 \\
1 & 3 & 2 & 4 & 1 & 4 & 2 & 3 & 2 & 3 & 4 & 1 & 4 & 3 & 2 & 1 \\
3 & 2 & 1 & 3 & 4 & 2 & 2 & 1 & 3 & 4 & 4 & 2 & 1 & 3 & 4 & 1 \\
3 & 3 & 4 & 3 & 4 & 2 & 3 & 4 & 2 & 1 & 4 & 2 & 1 & 2 & 1 & 1 \\
1 & 2 & 3 & 4 & 1 & 4 & 3 & 2 & 3 & 2 & 4 & 1 & 4 & 2 & 3 & 1 \\
4 & 2 & 2 & 1 & 1 & 1 & 3 & 3 & 3 & 3 & 4 & 4 & 4 & 2 & 2 & 1 \\
2 & 3 & 1 & 2 & 4 & 3 & 3 & 1 & 2 & 4 & 4 & 3 & 1 & 2 & 4 & 1 \\
4 & 4 & 1 & 4 & 1 & 4 & 4 & 1 & 4 & 1 & 1 & 4 & 1 & 4 & 1 & 1 \\
3 & 1 & 2 & 2 & 4 & 3 & 4 & 3 & 1 & 2 & 1 & 2 & 4 & 4 & 3 & 1 \\
2 & 1 & 3 & 3 & 4 & 2 & 4 & 2 & 1 & 3 & 1 & 3 & 4 & 4 & 2 & 1 \\
1 & 4 & 4 & 1 & 1 & 1 & 4 & 4 & 4 & 4 & 1 & 1 & 1 & 4 & 4 & 1
\end{array}\right] \cdot\left(\begin{array}{c}
\beta_{11} \\
\beta_{21} \\
\beta_{12} \\
\beta_{31} \\
\beta_{22} \\
\beta_{13} \\
\beta_{41} \\
\beta_{32} \\
\beta_{23} \\
\beta_{14} \\
\beta_{42} \\
\beta_{33} \\
\beta_{24} \\
\beta_{43} \\
\beta_{34} \\
\beta_{44}
\end{array}\right)=\left(\begin{array}{l}
1 \\
2 \\
3 \\
4 \\
2 \\
4 \\
1 \\
3 \\
3 \\
1 \\
4 \\
2 \\
4 \\
3 \\
2 \\
1
\end{array}\right)
$$


The rank of the matrix is equal to 16 , therefore it has the unique solution: $\beta_{11}=1$, and all the remaining coefficients $\beta_{i j}$ are equal to zero. Therefore,

$$
f_{5}^{(1,2)}(x, y)=x y
$$

In a similar way, we get that $f_{r}^{(1,2)}(x, y)=0$ for all $r \neq 5$.

Thus,

$$
a_{2}^{y} a_{1}^{x}=(x, y, 0,0, x y, 0,0,0,0,0,0,0,0,0,0,0) .
$$

Using this method, let's calculate other noncommutative pairs $a_{j}^{y} a_{i}^{x}$.

$$
\begin{aligned}
& a_{3}^{y} a_{1}^{x}=(x, 0, y, 0,0, x y, 0,0,0,0,0,0,0,0,0), \\
& a_{3}^{y} a_{2}^{x}=(0, x, y, 0,4 x y, 4 x y, 0,0,0,2 x y, 4 x y, 2 x y, 3 x y, 3 x y, x y), \\
& a_{4}^{y} a_{1}^{x}=\left(x, 0,0, y, 0,0, x y, 0,0,2 x y+3 x y^{2}, 0,0,2 x y+2 x y^{2}+x y^{3}, 0,0\right), \\
& a_{4}^{y} a_{2}^{x}=\left(0, x, 0, y, 0,0,0, x y, 0,0,2 x y+3 x y^{2}, 0,0,2 x y+2 x y^{2}+x y^{3}, 0\right) \text {, } \\
& a_{4}^{y} a_{3}^{x}=\left(0,0, x, y, 0,0,0,0, x y, 0,0,2 x y+3 x y^{2}, 0,0,2 x y+2 x y^{2}+x y^{3}\right), \\
& a_{5}^{y} a_{4}^{x}=(0,0,0, x, y, 0,0,0,0,0,0,0,2 x y, 0,0), \\
& a_{6}^{y} a_{4}^{x}=(0,0,0, x, 0, y, 0,0,0,0,0,0,0,4 x y, 0), \\
& a_{7}^{y} a_{2}^{x}=(0, x, 0,0,0,0, y, 0,0,0,0,0, x y, 0,0), \\
& a_{7}^{y} a_{3}^{x}=(0,0, x, 0,0,0, y, 0,0,0,0,0,0,2 x y, 0), \\
& a_{7}^{y} a_{4}^{x}=\left(0,0,0, x, 0,0, y, 0,0, x y, 0,0,2 x y+3 x^{2} y, 0,0\right), \\
& a_{8}^{y} a_{1}^{x}=(x, 0,0,0,0,0,0, y, 0,0,0,0,4 x y, 0,0), \\
& a_{8}^{y} a_{3}^{x}=(0,0, x, 0,0,0,0, y, 0,0,0,0,0,0, x y), \\
& a_{8}^{y} a_{4}^{x}=\left(0,0,0, x, 0,0,0, y, 0,0, x y, 0,0,2 x y+3 x^{2} y, 0\right), \\
& a_{9}^{y} a_{1}^{x}=(x, 0,0,0,0,0,0,0, y, 0,0,0,0,3 x y, 0), \\
& a_{9}^{y} a_{2}^{x}=(0, x, 0,0,0,0,0,0, y, 0,0,0,0,0,4 x y), \\
& a_{9}^{y} a_{4}^{x}=\left(0,0,0, x, 0,0,0,0, y, 0,0, x y, 0,0,2 x y+3 x^{2} y\right), \\
& a_{10}^{y} a_{4}^{x}=(0,0,0, x, 0,0,0,0,0, y, 0,0, x y, 0,0), \\
& a_{11}^{y} a_{4}^{x}=(0,0,0, x, 0,0,0,0,0,0, y, 0,0, x y, 0), \\
& a_{12}^{y} a_{4}^{x}=(0,0,0, x, 0,0,0,0,0,0,0, y, 0,0, x y) .
\end{aligned}
$$

Not listed pairs are commutative, i.e. $a_{j}^{y} a_{i}^{x}=a_{i}^{x} a_{j}^{y}$.

Thus, we have a complete set of relations for the implementation of the collection process in analytical form:

$$
a_{j}^{y} a_{i}^{x}=a_{i}^{x} a_{j}^{y} a_{j+1}^{f_{j+1}^{(i, j)}(x, y)} a_{j+2}^{f_{j+2}^{(i, j)}(x, y)} \ldots a_{15}^{f_{15}^{(i, j)}(x, y)}, \quad 1 \leqslant i<j \leqslant 15 .
$$

Using (17) we can calculate the product $a_{1}^{x_{1}} \ldots a_{15}^{x_{15}} \cdot a_{1}^{y_{1}} \ldots a_{15}^{y_{15}}=a_{1}^{z_{1}} \ldots a_{15}^{z_{15}}$. Following this procedure, we will find all $z_{i}(1-15)$. 


\section{Computer calculations of the growth of the group $\mathrm{C}$}

The calculation of the growth function of the group $C_{0}$ in alphabets $X_{0}=\left\{a_{1}, a_{2}, a_{3}, a_{4}\right\}$ and $Y_{0}=X_{0} \cup X_{0}^{-1}$ was carried out according to the algorithm from [10]. For efficient multiplication of elements Hall's polynomials obtained in section 2 were used. The algorithm was implemented in $\mathrm{C}++$. As a tool for parallelization, it was used OpenMP library. For the calculations, it was used the computer which has 4-core processor and 32 GB of RAM, running the Linux operating system. The program was compiled by the embedded compiler GCC. Calculating growth functions for the generating set $X_{0}$ takes about 1.5 hours, and for $Y_{0} 3$ hours.

Then it is easy to get the growth function of the group $C$ in alphabets generating $X=$ $\left\{a_{1}, a_{2}, a_{3}, a_{4}, z\right\}$ and $Y=X \cup X^{-1}$. Their graphs are shown in Figs. 1,2. For clarity, an approximating Gaussian curve obtained by the method of the least squares is added on each graph.

As already mentioned, the growth function of the group that containing information about the characteristics of the corresponding Cayley graph is:

Corollary 1. $D_{X}(C)=29, \bar{D}_{X}(C) \approx 21$.

Corollary 2. $D_{Y}(C)=19, \bar{D}_{Y}(C) \approx 14$.

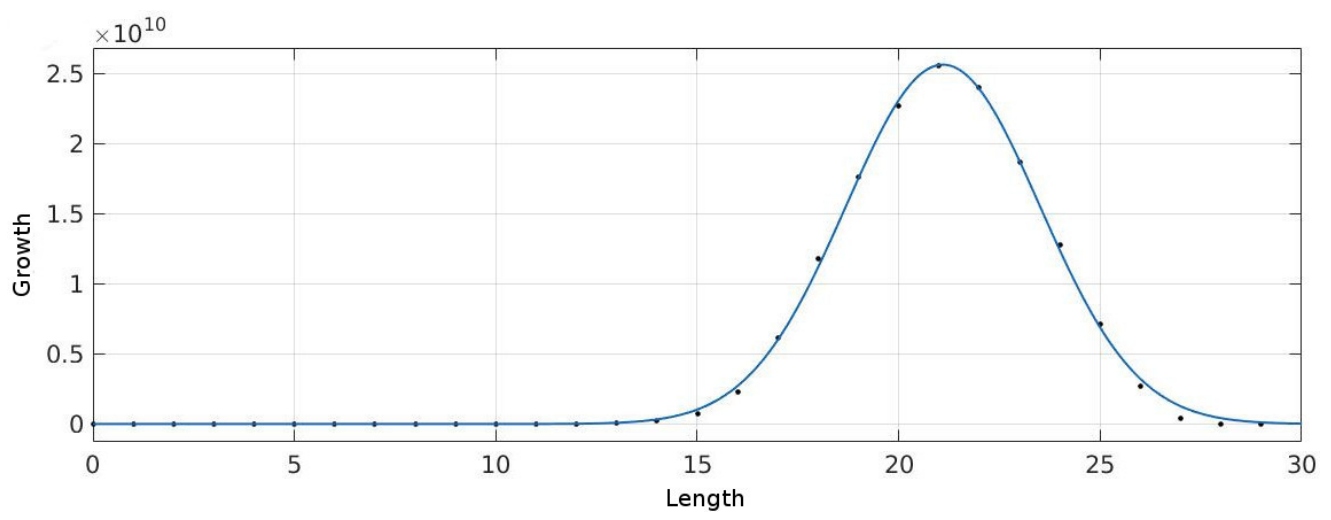

Fig. 1. The growth function of $C$ generated by $X$

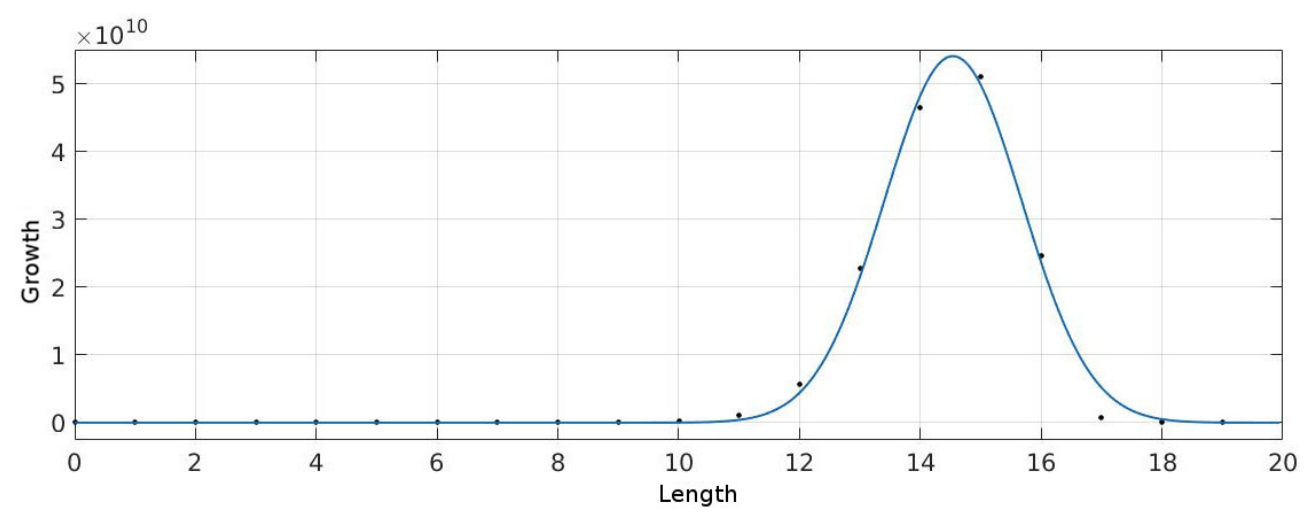

Fig. 2. The growth function of $C$ generated by $Y$ 
The reported study was funded by Russian Foundation for Basic Research, Government of Krasnoyarsk Territory, Krasnoyarsk Region Science and Technology Support Fund to the research project no. 17-47-240318.

\section{References}

[1] S.Even, O.Goldreich, The Minimum Length Generator Sequence is NP-Hard, J. of Algorithms, 2(1981), no. 3, 311-313.

[2] A.A.Kuznetsov, A.S.Kuznetsova, A parallel algorithm for study of the Cayley graphs of permutation groups, Vestnik SibGAU, 53(2014), no. 1, 34-39 (in Russian).

[3] S.Akers, B.Krishnamurthy, A group theoretic model for symmetric interconnection networks, Proceedings of the International Conference on Parallel Processing, (1986), 216-223.

[4] D.Holt, B.Eick, E.O'Brien, Handbook of computational group theory, Boca Raton, Chapman \& Hall/CRC Press.

[5] M.Camelo, D.Papadimitriou, L.Fàbrega, P.Vilà, Efficient Routing in Data Center with Underlying Cayley Graph, Proceedings of the 5th Workshop on Complex Networks Comple Net, 2014, 189-197.

[6] A.A.Kuznetsov, The Cayley graphs of Burnside groups of exponent 3, Sib. Elektron. Math. Izv., 12(2015), 248-254 (in Russian).

[7] A.A.Kuznetsov, A.S.Kuznetsova, Perspective topologies of multiprocessor computing systems based on the Cayley graphs of groups of period 4, Vestnik SibGAU, 17(2016), no. 3, 34-39 (in Russian).

[8] G. Havas, G.Wall, J. Wamsley, The two generator restricted Burnside group of exponent five, Bull. Austral. Math. Soc., 10(1974), 459-470.

[9] C.Sims, Computation with finitely presented groups, Cambridge, Cambridge University Press, 1994.

[10] A.A.Kuznetsov, An algorithm for computation of the growth functions in finite twogenerated groups of exponent 5, Prikl. Diskr. Math., 33(2016), no. 3, 116-125 (in Russian).

[11] A.A.Kuznetsov, K.A.Filippov, On an involutive automorphism of the Burnside group $B_{0}(2,5)$, Sib. Zh. Ind. Math., 13(2010), no. 3, 68-75 (in Russian).

[12] V.P.Shunkov, On periodic groups with almost regular involution, Algebra i Logika, 11(1972), no. 4, 470-494 (in Russian).

[13] Ph.Hall, Nilpotent groups, Notes of lectures given at the Canadian Mathematical Congress 1957 Summer Seminar, in The collected works of Philip Hall, Oxford, Clarendon Press, 1988, 415-462.

[14] A.A.Kuznetsov, A.S.Kuznetsova, Fast multiplication in finite two-generated groups of exponent five, Prikl. Diskr. Math., 18(2013), no. 1, 110-116 (in Russian). 
[15] A.A.Kuznetsov, K.V.Safonov, Hall's polynomials of finite two-generator groups of exponent seven, J. Sib. Fed. Univ. Math. Phys, 7(2014), no. 2, 186-190.

\title{
О приложениях графов Кэли некоторых конечных групп периода 5
}

\begin{abstract}
Александр А. Кузнецов
Константин В. Сафонов

Институт информатики и телекоммуникаций Сибирский государственный университет науки и технологий им. М. Ф. Решетнева Красноярский рабочий, 31, Красноярск, 660037

Россия

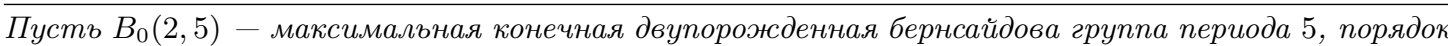
которой равен $5^{34}$. Определим автоморфизм $\varphi$, который инвертирует порождающие элементы. Пусть $C_{B_{0}(2,5)}(\varphi)$ - централизатор $\varphi$ в $B_{0}(2,5)$. Известно, что $\left|C_{B_{0}(2,5)}(\varphi)\right|=5^{16}$. В настоящей работе вычислены функции роста данного иентрализатора для некоторых порождающих множеств. В результате были получены диаметры и средние диаметры соответствующих графов Кэли $C_{B_{0}(2,5)}(\varphi)$.

Ключевые слова: периодическая группа, собирательный прочесс, полиномы Холла, граф Кэли, многопрочессорная вычислительная система.
\end{abstract}

